\title{
Les paléoenvironnements sédimentaires au Wisconsinien moyen et supérieur, île d'Anticosti, golfe du Saint-Laurent, Québec
}

\author{
Sedimentary Paleoenvironments during the Mid- and \\ Late-Wisconsinan \\ Paläogeographische Umwelt der Sedimente im mittleren und \\ späteren Wisconsin auf der Insel Anticosti, Sankt Lorenz-Golf, \\ Québec
}

\author{
Denis Gratton, Q. H. J. Gwyn et J. M. M. Dubois
}

Volume 38, numéro 3, 1984

URI : https://id.erudit.org/iderudit/032565ar

DOI : https://doi.org/10.7202/032565ar

\section{Aller au sommaire du numéro}

Éditeur(s)

Les Presses de l'Université de Montréal

ISSN

0705-7199 (imprimé)

1492-143X (numérique)

Découvrir la revue

Citer cet article

Gratton, D., Gwyn, Q. H. J. \& M. Dubois, J. M. (1984). Les paléoenvironnements sédimentaires au Wisconsinien moyen et supérieur, île d'Anticosti, golfe du Saint-Laurent, Québec. Géographie physique et Quaternaire, 38(3), 229-242. https://doi.org/10.7202/032565ar

\section{Résumé de l'article}

L'inlandsis laurentidien n'a jamais dépassé la côte sud de l'île d'Anticosti ni pénétré dans le golfe du Saint-Laurent après 36000 ans BP. De plus, la corrélation entre les unités stratigraphiques, accompagnée de datations au $14 \mathrm{C}$, permet la description des événements liés à trois avancées glaciaires du Quaternaire supérieur au centre-sud de l'île. En premier lieu, dans les vallées inondées par la transgression marine, l'avènement d'une masse de glace, au Wisconsinien moyen, met en place des dépôts glacio-marins datés de 36000 ans BP. Par contre. Ia glace s'étend sur les interfluves élevés adjacents et abandonne un till daté de 30000 ans BP. À la limite des secteurs côtiers, le vêlage intensif du front glaciaire provoque la mise en place, sur les côtes basses, de sédiments glacio-marins datés de 28000 ans BP, définissant par le fait même les variations importantes de la dynamique d'écoulement glaciaire dans un milieu marin. En deuxième lieu, une récurrence au Wisconsinien supérieur s'arrête à la côte sud de l'île, comme en témoigne la présence, dans les embouchures de vallées et dans la zone côtière, de sédiments glacio-marins datés de 13500 ans BP. La mise en place de cette deuxième unité de matériel glacio-marin confirme les perturbations importantes du front glaciaire que provoque à cette latitude une grande profondeur d'eau. En troisième lieu, lors de la phase de régression marine, une calotte résiduelle qui ne couvre que les interfluves et les vallées secondaires avance à la limite de la zone côtière et met en place un matériel glacio-marin daté de 12000 ans BP. Cette récurrence est le résultat d'un réajustement gravitationnel relié au relèvement isostatique ainsi qu'à l'abaissement du niveau marin.
Tous droits réservés @ Les Presses de l'Université de Montréal, 1984
Ce document est protégé par la loi sur le droit d'auteur. L’utilisation des services d'Érudit (y compris la reproduction) est assujettie à sa politique d'utilisation que vous pouvez consulter en ligne.

https://apropos.erudit.org/fr/usagers/politique-dutilisation/ 


\section{LES PALÉOENVIRONNEMENTS SÉDIMENTAIRES AU WISCONSINIEN MOYEN ET SUPÉRIEUR, ÎLE D’ANTICOSTI, GOLFE DU SAINT-LAURENT, QUEBEC}

Denis GRATTON, Q.H.J. GWYN et J.M.M. DUBOIS, Département de géographie, Université de Sherbrooke, Sherbrooke, Québec J1K 2R1.

RÉSUMÉ L'inlandsis laurentidien n'a jamais dépassé la côte sud de l'île d'Anticosti ni pénétré dans le golfe du Saint-Laurent après 36000 ans BP. De plus, la corrélation entre les unités stratigraphiques, accompagnée de datations au ${ }^{14} \mathrm{C}$, permet la description des événements liés à trois avancées glaciaires du Quaternaire supérieur au centre-sud de l'île. En premier lieu, dans les vallées inondées par la transgression marine, l'avènement d'une masse de glace, au Wisconsinien moyen, met en place des dépôts glacio-marins datés de 36000 ans BP. Par contre, la glace s'étend sur les interfluves élevés adjacents et abandonne un till daté de 30000 ans BP. À la limite des secteurs côtiers, le vêlage intensif du front glaciaire provoque la mise en place, sur les côtes basses, de sédiments glacio-marins datés de 28000 ans BP, définissant par le fait même les variations importantes de la dynamique d'écoulement glaciaire dans un milieu marin. En deuxième lieu, une récurrence au Wisconsinien supérieur s'arrête à la côte sud de lîle, comme en témoigne la présence, dans les embouchures de vallées et dans la zone côtière, de sédiments glacio-marins datés de 13500 ans BP. La mise en place de cette deuxième unité de matériel glacio-marin confirme les perturbations importantes du front glaciaire que provoque à cette latitude une grande profondeur d'eau. En troisième lieu, lors de la phase de régression marine, une calotte résiduelle qui ne couvre que les interfluves et les vallées secondaires avance à la limite de la zone côtière et met en place un matériel glacio-marin daté de 12000 ans BP. Cette récurrence est le résultat d'un réajustement gravitationnel relié au relèvement isostatique ainsi qu'à l'abaissement du niveau marin.
ABSTRACT Sedimentary paleoenvironments during the mid-and late-Wisconsinan, Anticosti Island, Gulf of St. Lawrence, Québec. The Laurentide Ice Sheet did not extend beyond the south coast of Anticosti Island into the Gulf of St. Lawrence after 36000 years BP. Correlation of stratigraphic units, supported by ${ }^{14} \mathrm{C}$ dates, reveal the events associated with three glacial advances during the late Quaternary of the south-central part of the island. Firstly, during the midWisconsinan the ice sheet advanced into drowned valleys and deposited glacial-marine sediments dated at $36000 \mathrm{yrs}$ BP. The ice also covered adjacent interfluves and deposited a till dated at 30000 yrs BP. Subsequently, in the present coastal zones, intense calving at the ice front resulted in the deposition of glacial-marine sediments dated at 28000 yrs BP. This illustrates the important variations in the flow dynamic of the ice sheet in a marine environment. Secondly, during the late-Wisconsinan the ice sheet readvanced again to the south coast of the island but did not extend into the Gulf. This is revealed by the presence of glacial-marine sediments, dated at $13500 \mathrm{yrs} B P$, at the mouths of river valleys and in the actual low coastal zones. Deposition of this second glacial-marine unit confirmes the influence, at this latitude, of a deep water marine environment on the extension of the glacial front. Thirdly, contemporaneously with the marine regression, a residual ice cap that covered the interfluves and certain small valleys advanced to the limit of the present coastal zone and deposited glacial-marine sediments dated at 12000 yrs BP. This advance resulted from gravitational reajustments as a function of the crustal uplift and the lowered marine limit.
ZUSAMMENFASSUNG Paläogeographische Umwelt der Sedimente im mittleren und späteren Wisconsin auf der Insel Anticosti, Sankt Lorenz-Golf, Québec. Das Laurentische Inlandeis ist niemals über die Südküste der Insel Anticosti hinausgekommen, noch ist es in den Golf des Sankt Lorenz-Stroms nach 36000 v.u.Z. eingedrungen. In den von der Meer-Transgression überschwemmten Tälern hat das Vordringen einer Eismasse im mittleren Wisconsin glazial-marine Ablagerungen hinterlassen, die auf 36000 Jahre v.u.Z. datiert werden. Andererseits hat sich das Eis bis auf die erhöhten angrenzenden Interfluves ausgedehnt und Geschiebelehm abgelagert, der auf 30000 Jahre v.u.Z. datiert wird. An der Grenze der Küstengebiete hat das intensive Kalben der Eisfront die Ablagerung von glazial-marinen Sedimenten an den tiefliegenden Küsten hervorgerufen, die auf 28000 Jahre v.u.Z. datiert werden. Hierdurch werden die erheblichen Variationen in der StrömungsDynamik der Eisdecke im Meeres-Milieu itlustriert. Zweitens hält ein Vorsto ß im späten Wisconsin an der Südküste der Insel an, wie es durch glazial-marine Sedimente bestätigt wird, die sich in den Talmündungen und im Küstengebiet befinden und die auf 13500 Jahre v.u.Z. datiert werden. Die Ablagerung dieser zweiten Einheit von glazial-marinem Material bestätigt die erheblichen Störungen der Eisfront, die in dieser Zone durch eine beträchtliche Wassertiefe verursacht werden. Drittens stößt während der Phase des Sinkens des Meeresspiegels eine Restkalotte, die nur die Interfluves und die Sekundärtäler bedeckt, bis zur Grenze des Küstengebiets vor und lagert glazial-marines Material ab, das auf 12000 Jahre v.u.Z. datiert wird. Dieser Vorsto $\beta$ ist das Ergebnis einer Schwere-Wiederanpassung, in Verbindung mit einer isostatischen Anhebung und dem Sinken des Meeresspiegels. 


\section{INTRODUCTION}

L'extension et la dynamique d'écoulement de l'inlandsis laurentidien, au Wisconsinien supérieur dans la région du golfe du Saint-Laurent (fig. 1), sont à la source d'une polémique opposant deux écoles de pensée. L'hypothèse maximaliste, présentée par FLINT (1943) et soutenue récemment par DENTON et HUGHES (1981), fait intervenir une épaisseur importante de glace dont la dynamique d'écoulement permet une extension évidente dans le golfe. En définitive, seules les grandes profondeurs d'eau, d'au-delà de la plate-forme continentale, ont pu arrêter l'extension de la glace. Par conséquent, selon eux, l'île d'Anticosti aurait été entièrement englacée jusqu'à l'Holocène.

GRANT $(1976,1977)$ de son côté, reprend des idées déjà émises et élabore l'hypothèse d'une couverture minimale de glace. II définit l'île d'Anticosti comme un nunatak et propose la présence de calottes indépendantes à Terre-Neuve et en
Nouvelle-Écosse. IVES (1978) favorise lui aussi un englacement limité des zones côtières avec des calottes locales couvrant les provinces atlantiques et une glace flottante dans le chenal laurentien. Enfin, une visite sur le terrain en 1977 suffit à l'un de nous pour le convaincre qu'au moins la partie ouest de lîle avait été englacée au cours du dernier événement glaciaire (DUBOIS, 1979).

Récemment, PAINCHAUD, DUBOIS et GWYN $(1982,1984)$ ont décrit les événements liés à la période fini-glaciaire du Wisconsinien supérieur dans la partie ouest de l'île d'Anticosti. Deux conclusions majeures s'imposent: un englacement certain de l'ouest de l'île au Wisconsinien supérieur et l'abandon d'une calotte résiduelle centrale lors de la déglaciation. Cette calotte résiduelle est délimitée au sud par un bourrelet morainique et au nord et à l'ouest, par des épandages proglaciaires et quelques fronts morainiques. PAINCHAUD, DUBOIS et GWYN $(1982,1984)$ situent ces événements entre 13000 et 11500 ans BP.

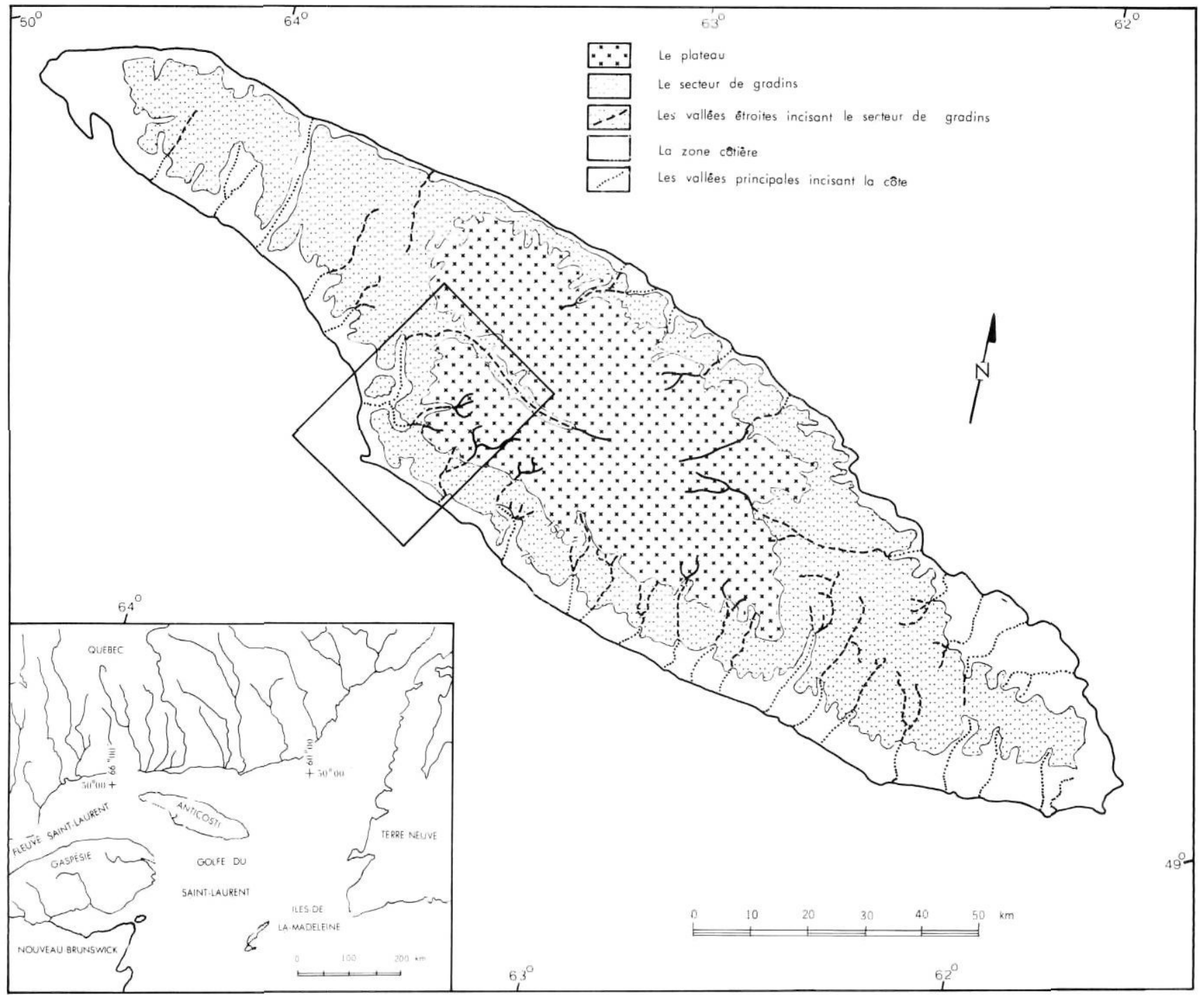

FIGURE 1. Carte de localisation et schéma géomorphologique de l'île d'Anticosti; délimitation de la région à l'étude.
Location map and regional geomorphological sketch; location of study area. 
Poursuivant ces travaux, cet article décrit la lithostratigraphie du centre-sud de l'île (fig 1), en mettant l'accent sur huit successions d'unités stratigraphiques à l'intérieur de quatre unités géomorphologiques. À chaque unité géomorphologique correspondent des environnements de sédimentation distincts. À partir de cette étude, on reconstitue l'histoire du Quaternaire récent de la région étudiée et on répond à la question controversée de l'extension des glaces dans le golfe, au sud de l'île d'Anticosti.

\section{DESCRIPTION DES UNITÉS GÉOMORPHOLOGIQUES}

Le substratum de l'île d'Anticosti est constitué de couches de calcaires paléozoïques ayant un pendage de moins de cinq degrés vers le sud-ouest (PÉTRYK, 1979). Cet agencement influence le relief et permet une division de l'île en quatre unités géomorphologiques principales (fig. 1). La région étudiée est délimitée par la rivière Jupiter à l'ouest et au nord et par la rivière du Brick à l'est (fig. 2).

La première unité géomorphologique est constituée d'un plateau peu disséqué de plus de $150 \mathrm{~m}$ d'altitude (fig. 2). La météorisation mécanique et chimique des calcaires est responsable de la présence de multiples dolines dont plusieurs sont inondées. Sur les rebords du plateau, certaines de ces dépressions sont ouvertes sur de petits ravins qui représentent la tête du réseau hydrographique.

La deuxième unité géomorphologique est constituée d'une série de gradins successifs morcelée de vallées étroites et profondes et localisée entre le rebord du plateau et $75 \mathrm{~m}$ d'altitude environ (fig. 2). La présence des gradins est reliée à l'érosion différentielle du substratum rocheux. Ils ne sont pas d'égale hauteur. Leurs surfaces, relativement planes, sont partiellement recouvertes de dépôts meubles. Leurs versants rocheux ont une pente de l'ordre de 5 à $10^{\circ}$. Les talwegs des vallées sont généralement rectilignes lorsqu'ils sont taillés dans le substratum rocheux, mais sinueux lorsqu'ils sont incisés dans du matériel meuble. Les ravins, profonds de $50 \mathrm{~m}$ en moyenne, ont soit des versants rocheux avec des pentes supérieures à 45 degrées, soit des versants moins abrupts de 30 degrés, dans des dépôts meubles.

La troisième unité géomorphologique est constituée par les trois vallées principales qui s'évasent à une altitude de moins de $75 \mathrm{~m}$, altitude à laquelle les vallées étroites s'intègrent à ces axes majeurs (fig. 2). Les cours d'eau sont plus sinueux que ceux de la deuxième unité puisqu'ils coulent sur des dépôts meubles. Les versants ont des pentes inférieures à 45 degrés et, s'ils ne sont pas constitués de dépôts meubles, ils sont formés d'une succession de paliers rocheux. À moins de $5 \mathrm{~km}$ des embouchures, la pente des rivières diminue, résultat d'un comblement important du plancher des vallées au cours des processus de déglaciation et de régression marine.

La dernière unité géomorphologique est le secteur côtier (fig. 2). Sa géomorphologie est influencée par la présence de gradins qui donnent au littoral une double apparence: une côte haute, formée par le rebord des gradins, avec une pente allant de $45^{\circ}$ à $90^{\circ}$ et une hauteur de 20 à $75 \mathrm{~m}$ et une côte basse d'une hauteur inférieure à $20 \mathrm{~m}$, formée par la surface des gradins et souvent adoucie par des épandages importants de dépôts meubles. Dans le secteur d'étude, la côte basse, nommée la côte Verte, sépare deux côtes hautes: les brisants Jumper à l'est et le cap Ottawa à l'ouest.

\section{DESCRIPTIONS DES UNITÉS STRATIGRAPHIQUES}

La description des huit successions d'unités sédimentaires associées aux unités géomorphologiques (GWYN, DUBOIS, PAINCHAUD et GRATTON, 1982; GWYN, DUBOIS et GRATTON, 1983) permettra d'expliciter l'évolution géologique quaternaire du secteur (fig. 3).

\section{LE PLATEAU}

Sur le plateau, on ne retrouve qu'une unité stratigraphique. Les couvertures importantes de matériaux meubles sont rares. C'est seulement à l'intérieur de quelques dépressions qu'apparaît un till silto-argileux brun pâle (10YR7/3) d'une épaisseur variable, mais dépassant rarement le mètre (fig. 3 , colonnes $A$ et $A^{\prime}$, tabl. I). Le till contient un minimum de $15 \%$ de galets, souvent striés, dans une matrice dont la teneur en carbonates est de l'ordre de $42,8 \%$ à $80,4 \%$. Ce till ne contient que quelques traces de minéraux lourds (moins de $0,01 \%$ dans les sables fins); cette caractéristique se retrouve dans la totalité des échantillons de till et de matériel glacio-marin prélevés dans la région à l'étude. II y a donc eu une très forte contribution locale et un faible apport du bouclier Canadien.

\section{LE SECTEUR DE GRADINS ET DE VALLÉES ÉTROITES}

Sur les interfluves en gradins (fig. 3, colonne B, tabl. I), on trouve deux tills: un till brun pâle (10YR7/3) et un till gris pâle avec des fragments de coquillages (7,5YR7/0). Les tills sont silto-argileux, bien que le second soit plus argileux (c'està-dire $28,6 \%$ en moyenne, contre $22,0 \%$ ), et ont de fortes teneurs en carbonates $(55,2 \%$ à $84,4 \%)$ (tabl. I).

Les vallées présentent des versants rocheux pré-glaciaires couverts par endroits par des placages de till et de gravier fluviatile (fig. 3 , colonne C). Le till brun pâle (10YR7/3) est aussi silto-argileux et compact. II contient $20 \%$ de galets subanguleux dont $95 \%$ sont calcaires. Le pourcentage de carbonates dans la matrice se situe entre $65,6 \%$ et $78,7 \%$. La présence du till est plus continue dans la vallée de la rivière Jupiter où il peut atteindre des épaisseurs de plus de $9 \mathrm{~m}$. Un gravier sableux à couches entrecroisées vers le sud couvre le till. D'origine fluviatile, le gravier date de la période d'exondation.

\section{LES VALLÉES PRINCIPALES INCISANT LA CÔTE}

Les vallées principales contiennent des dépôts qui constituent la transition lithologique entre le secteur côtier et le secteur de plateau. Cette unité géomorphologique montre une série d'unités sédimentaires interreliant les milieux marin et glaciaire (GRATTON et al. 1982; GWYN, GRATTON et DUBOIS, 1983). 


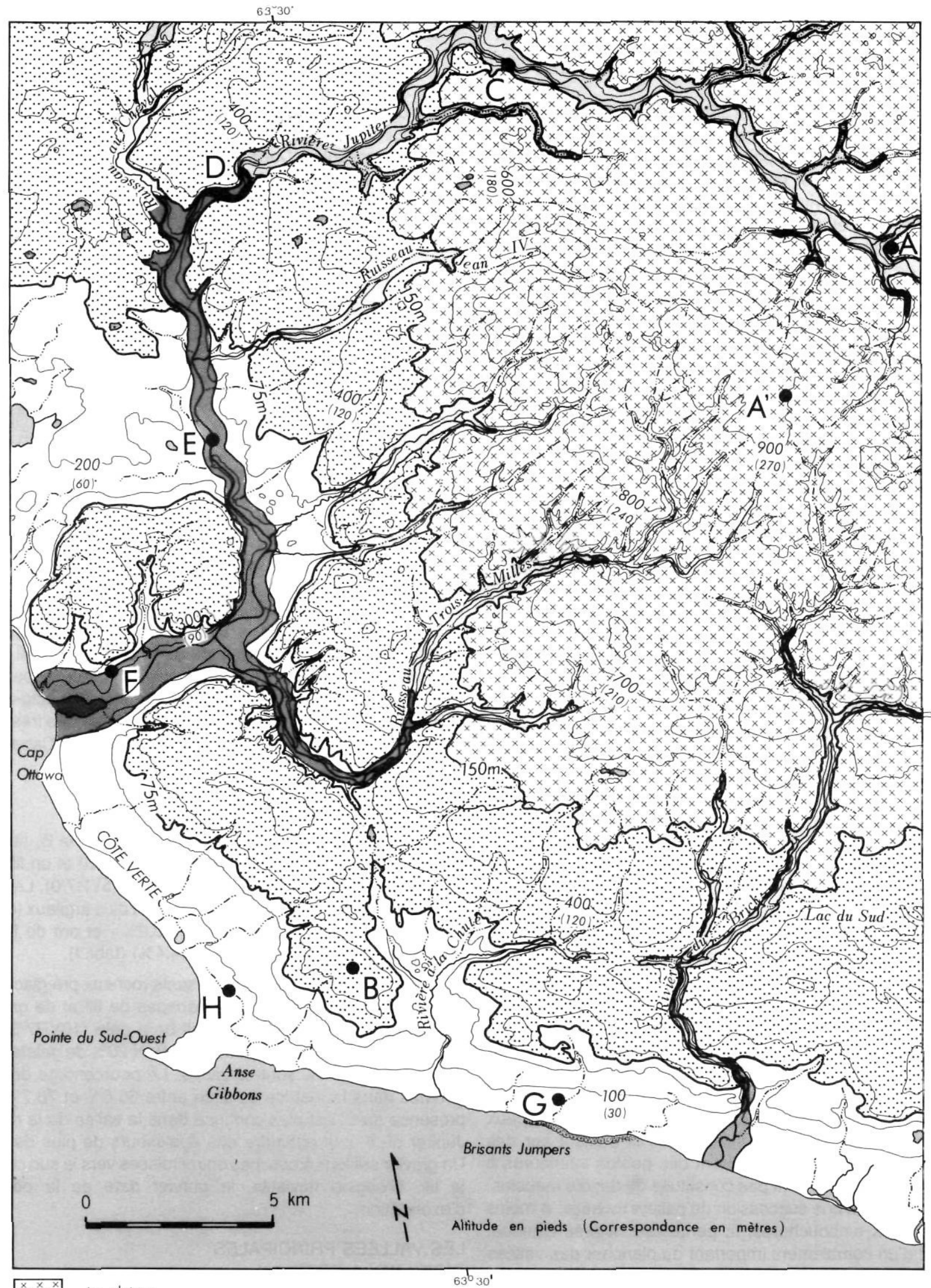

$\left[\begin{array}{l}x x^{x} x^{x} x \\ x x^{x}\end{array} \quad\right.$ Le plateau

$\because \cdots:$ Le secteur de gradins

Les vallées étroites incisant le secteur de gradins

Lo zone côtière

Les vallées principales incisant la côte
FIGURE 2. Toponymie et géomorphologie détaillée de la région à l'étude; localisation de la succession des unités stratigraphiques décrites à la figure 3 .

Place-names and detailed geomorphology of the studied area; location of stratigraphic sequences described at Figure 3.

H Localisation des colonnes décrites à la figure 3 
TABLEAU I

Résumé des caractéristiques granulométriques et les teneurs en carbonates des dépôts meubles, centre sud de l'île d'Anticosti

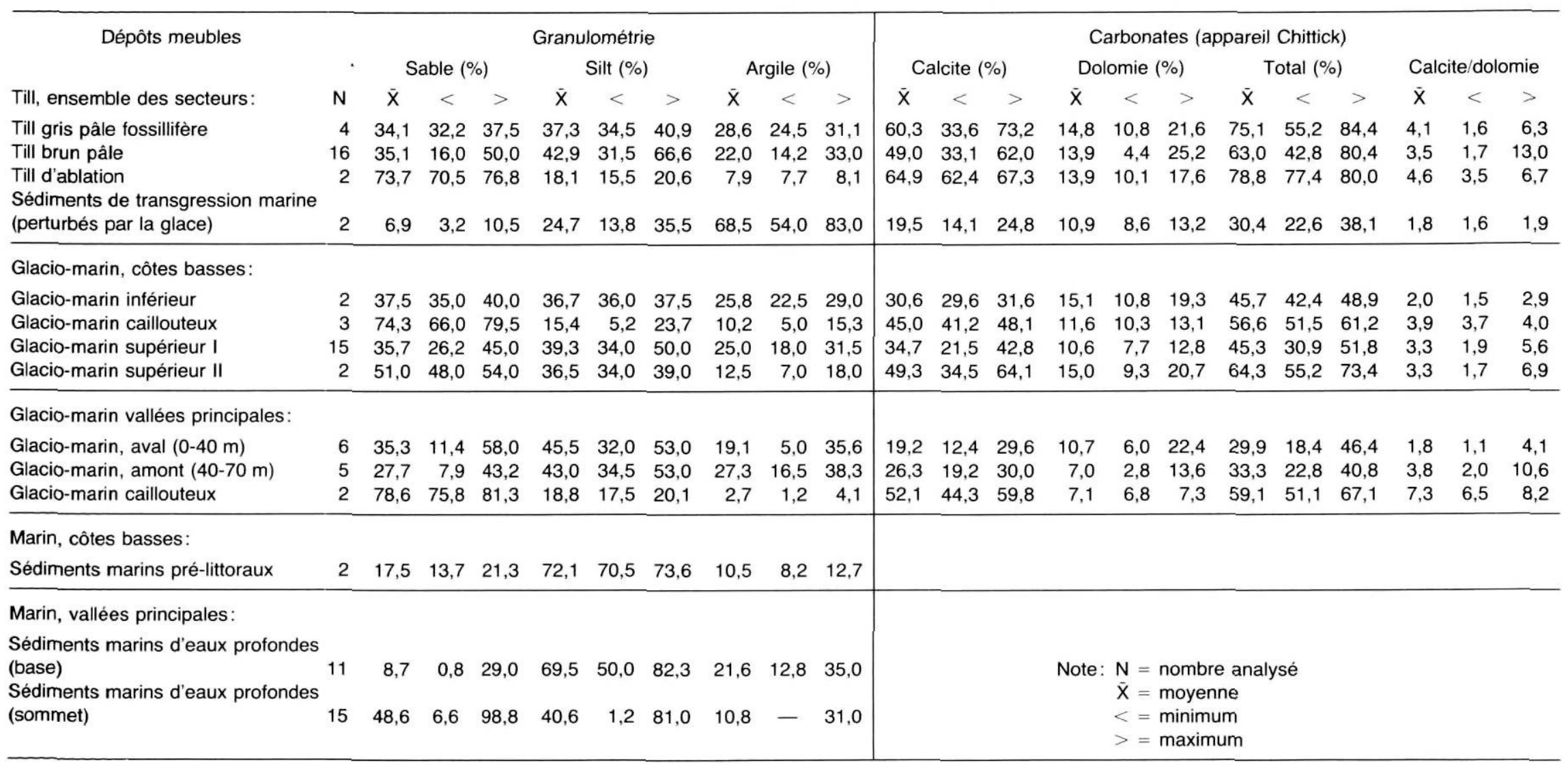


La stratigraphie à l'intérieur des vallées principales est complexe; cependant, on peut la diviser en trois successions d'unités stratigraphiques.

Première succession d'unités stratigraphiques: confluence des vallées étroites et des vallées principales

La première succession d'unités stratigraphiques est formée de cinq unités (fig. 3 , colonne $D$ et tabl. I):

1. La première unité est constituée de stratifications entrecroisées de type fluviatile composées de trois lits distincts totalisant environ $4 \mathrm{~m}$ d'épaisseur. De bas en haut, on trouve: 1) environ $1 \mathrm{~m}$ de sable caillouteux et de gravier sableux interlités; 2) environ $1 \mathrm{~m}$ de lamelles horizontales de sable et de silt; 3) $2 \mathrm{~m}$ de gravier sableux à structures entrecroisées dont les éléments sont imbriqués, indiquant un écoulement vers le sud.

2. La deuxième unité se caractérise par des sédiments lamellaires dont la composition passe du sable silteux vers le bas à l'argile silteuse vers le haut. Dans cette unité, les teneurs en carbonates décroissent de bas en haut, passant de $72,2 \%$ à $30,0 \%$. Ces sédiments ont probablement été mis en place au cours d'une inondation marine. Ils sont partiellement perturbés par des déformations glacio-tectoniques suggérant une avancée glaciaire vers le sud-est. En effet, incorporées à des rides d'écoulement, on trouve des lamelles inclinées dont le pendage est de $35^{\circ}$ vers le sud-est et la direction de $020^{\circ}$, ainsi que des lamelles plissées avec un axe de plis orientés à $100^{\circ}$. Dans la moitié supérieure de cette unité, on dénote la présence de galets anguleux et striés, de même que des fragments de coquillages.

3. La deuxième unité s'amincit en aval et on passe abruptement à la troisième unité qui est constituée d'un till siltoargileux brun pâle (10YR7/3) dont la teneur en carbonates atteint $64,3 \%$.

4. La quatrième unité est constituée d'une série de lamelles prodeltaïques, dont la composition passe du silt au sable vers le haut.

5. Au sommet de la quatrième unité, la granulométrie devient de plus en plus grossière et les lamelles de sable prodeltaïques laissent place à des sables caillouteux et à des graviers sableux interlités, liés à une phase fluviatile d'exondation. Ces sédiments constituent la cinquième unité.

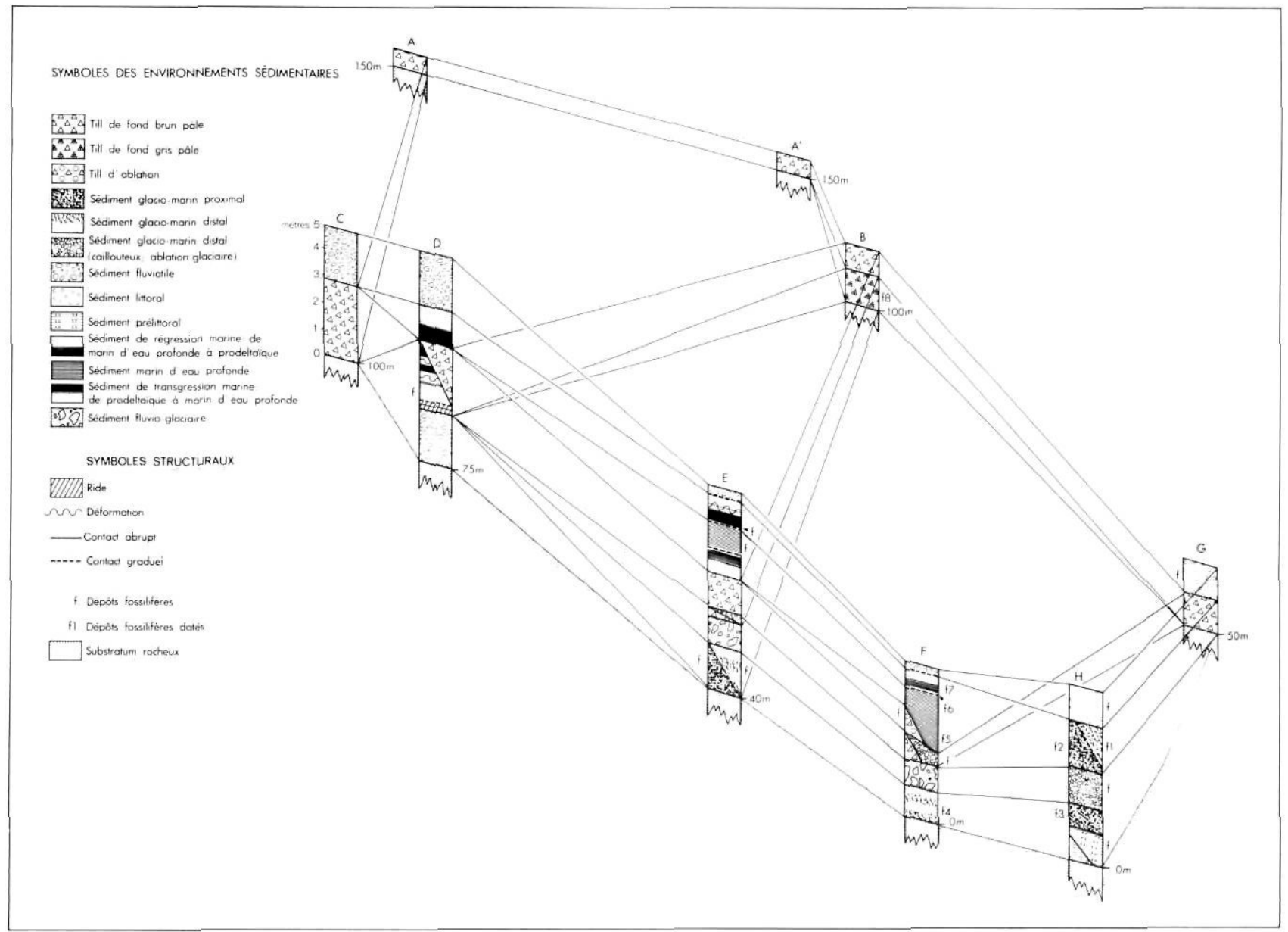

FIGURE 3. Description et corrélation des huit successions d'unités stratigraphiques; $\mathrm{f} 1$ à f8 réfèrent aux positions stratigraphiques des unités fossilifères datées et décrites au tableau II.
Description and correlation of the stratigraphic sequences; $f 1$ to $f 8$ refer to the stratigraphic position of dated fossiliferous units described in Table II. 


\section{Deuxième succession d'unités stratigraphiques: les vallées principales}

La deuxième succession d'unités stratigraphiques (fig. 3, colonne $\mathrm{E}$ et tabl. I) comprend sept unités:

1. La première unité est un matériel glacio-marin formé de deux lits interstratifiés dont le contact, d'amont en aval, est graduel (GWYN, GRATTON, PAINCHAUD, DAIGNEAULT et DUBOIS, 1982). Le premier lit, en amont, est représenté par plus de $2 \mathrm{~m}$ de silt argileux caillouteux compact, lenticulaire et fossilifère. Latéralement, cette unité possède des caractéristiques granulométriques variables: de $7,9 \%$ à $43,2 \%$ de sable à $5 \%$ à $10 \%$ de galets. Les galets sont fréquemment striés, mais leur émoussé varie d'arrondi à subanguleux. La teneur en carbonates varie entre $22,8 \%$ et $40,8 \%$. Le deuxième lit, également d'origine glacio-marine, est un silt fossilifère compact et lenticulaire, intercalé près de la surface de lentilles de silt sableux caillouteux également fossilifère. Les galets $(5 \%)$ sont striés. L'épaisseur de ce lit est généralement supérieure à $2 \mathrm{~m}$. Les teneurs en carbonates sont relativement faibles, car elles varient entre $18,2 \%$ et $46,4 \%$. Les deux dépôts glacio-marins sont différents du fait que le premier est granulométriquement homogène, tandis que le second est constitué de lentilles de texture variable.

2. La deuxième unité surmontant les dépôts glacio-marins de la vallée est un lit de matériel fluvio-glaciaire peu stratifié de plus de 3 m d'épaisseur, composé de galets mal triés avec moins de $15 \%$ de matrice de sable grossier. Les galets sont généralement sub-anguleux et plusieurs sont striés.

3. La deuxième unité est interstratifiée avec une troisième unité: un till silto-argileux brun pâle $(10 \mathrm{Y} 7 / 3)$ très caillouteux (25\%), atteignant $3 \mathrm{~m}$ d'épaisseur et dont la plupart des galets sont sub-anguleux. La teneur en carbonates est en moyenne de $65,4 \%$, mais elle peut varier entre $55,6 \%$ et $79,3 \%$.

4. La quatrième unité n'est présente qu'en amont des vallées principales, à une altitude supérieure à $40 \mathrm{~m}$. Elle est caractérisée par une structure lamellaire de sable et de silt de $1 \mathrm{~cm}$ d'épaisseur en moyenne, avec des rides indiquant un écoulement conforme à l'actuel. L'unité passe ensuite graduellement à des couches plus minces de silt et d'argile. Ce faciès représente une sédimentation de type prodeltaïque précédant une transgression marine rapide.

5. Par contre, à une altitude inférieure à $40 \mathrm{~m}$, la cinquième unité repose directement sur la troisième. Elle est composée d'un matériel silto-argileux fossilifère sédimenté en milieu marin d'eau profonde. L'apparence massive du matériel est probablement causée tant par l'intense floculation de la fraction argileuse en milieu glacio-marin proximal (OSTERMAN et ANDREWS, 1983) que par des phénomènes de bioturbation. Des lentilles de galets souvent striés, surtout à la base de l'unité, suggèrent la présence d'une certaine stratification. Vers le sommet, l'unité devient plus fossilifère et on remarque, entre autres, des Mya arenaria en position de vie. L'unité peut parfois atteindre $10 \mathrm{~m}$ d'épaisseur dans la vallée de la rivière Jupiter.

6. La sixième unité représente la phase de régression marine: des lamelles de silt fossilifère sont progressivement remplacées par des lamelles de sable fin et de sable grossier de plus en plus caillouteux et de moins en moins fossilifère. Cette unité suggère une sédimentation prodeltaïque lors de l'exondation marine. II faut noter que les silts à la base de cette unité sont déformés et que les sables grossiers caillouteux sont inclinés à $45^{\circ}$ : ce sont deux phénomènes structuraux indicateurs d'une perturbation, probablement glacio-tectonique, postérieure à la mise en place de la séquence de lamelles décrite précédemment.

7. La septième et dernière unité de la deuxième succession est représentée par $10 \mathrm{~m}$ de sédiments fluviaux. Ce sont des graviers et des blocs dans une matrice de sable mal trié, intercalés entre des couches de sable caillouteux avec des rides. Cette unité a été mise en place lors de la phase finale d'exondation.

Troisième succession d'unités stratigraphiques: embouchures des vallées principales

La troisième succession d'unités stratigraphiques identifiée dans les vallées principales se situe entre $20 \mathrm{~m}$ d'altitude et le niveau actuel de la mer (fig. 3, colonne F, tabl. I). Elle comprend sept unités dont certaines sont interstratifiées:

1. La première unité est formée de $2 \mathrm{~m}$ de sédiments glaciomarins fossilifères constitués de lentilles de silt compact intercalées entre des lentilles de silt sableux caillouteux. Ces lentilles contiennent $5 \%$ de galets striés, dont l'émoussé varie d'arrondi à sub-anguleux. La teneur moyenne en carbonates contenus dans la matrice varie entre $21,6 \%$ et $24,8 \%$.

2. La deuxième unité consiste en une unité de matériaux fluvio-glaciaires de plus de $3 \mathrm{~m}$ de gravier sableux mal trié à stratification peu marquée. Les galets de cette unité sont subanguleux et souvent striés.

Surmontant ces matériaux fluvio-glaciaires, on constate la présence de deux unités interstratifiées d'amont en aval, les troisième et quatrième unités.

3. La troisième unité est un till silto-argileux brun pâle (10YR7/ 3) de près de $5 \mathrm{~m}$ d'épaisseur dont la teneur en carbonates est de $65 \%$.

4. Plus près de l'embouchure, le till disparaît pour faire place à un dépôt glacio-marin caillouteux, la quatrième unité, très compacte et composée de galets et de graviers grossiers sub-anguleux et striés dans une matrice de sable très mal trié avec plusieurs fragments de coquillages.

5. La cinquième unité est formée d'un matériel juxta-glaciaire semblable à un till d'ablation. Ce matériel est massif et constitué de $50 \%$ de sable silteux et de $50 \%$ de galets et de blocs striés avec des fragments de coquillages. La teneur en carbonates varie entre $77,4 \%$ et $80,0 \%$.

6. La sixième unité couvre à la fois le till d'ablation et le matériel glacio-marin caillouteux. Massive, elle est constituée de sédiments marins d'eau profonde silto-argileux fossilifères. On remarque des zones de concentration de Mya truncata en position de vie et la présence, en particulier vers la base, de plus de $5 \%$ de galets striés sub-anguleux à sub-arrondis. 
7. La septième unité est un lit de silt et de sable prodeltaïques passant graduellement à un sable graveleux et un gravier sableux relié à la phase deltaïque et fluviatile d'exondation.

\section{LA ZONE CÔTIĖRE}

On distingue deux successions d'unités stratigraphiques dans la zone côtière; l'une associée aux secteurs de côte haute et l'autre associée au secteur de côte basse (fig. 3, colonne $\mathrm{G}$ et $\mathrm{H}$; tabl. I).

Dans les secteurs de la côte haute, soit sur le rebord des gradins, on observe des placages de till d'une épaisseur maximale de $2 \mathrm{~m}$ surmontés par un gravier de plage fossilifère souvent de plus de $5 \mathrm{~m}$ d'épaisseur. Le till, silto-argileux, est brun pâle (10YR $7 / 3)$ et contient entre $15 \%$ et $20 \%$ de galets striés. La teneur en carbonates est toujours supérieure à $65 \%$.

Dans le secteur de côte basse, comme la Côte Verte, le milieu est surtout glacio-marin. La base de la colonne stratigraphique montre une séquence marine prélittorale représentée par des rythmites de silt sous-jacentes à des lamelles de silt et de sable fin interstratifié contenant quelques fragments de coquillages. Cette unité varie latéralement de $0,75 \mathrm{~m}$ à $5,5 \mathrm{~m}$ d'épaisseur. Elle repose soit sur un sable caillouteux littoral ( $70 \%$ de galets) très compact qui couvre le substratum rocheux, soit directement sur le substratum rocheux.

La deuxième unité de la succession est formée de 1,75 m de matériel glacio-marin composé d'une série de lentilles silto-sableuses ou silto-argileuses caillouteuses. Sa structure est semblable à celle du matériel glacio-marin échantillonné dans la section amont des vallées principales (fig. 3 , colonne E), mais il est plus caillouteux (15\%) et plus fossilifère. La teneur en carbonates y est aussi un peu plus élevée, soit supérieure à $40 \%$.

Au-dessus de l'unité inférieure de matériel glacio-marin se trouve un dépôt glacio-marin caillouteux de plus de $7 \mathrm{~m}$ d'épaisseur constitué de galets souvent striés, enchassés dans une matrice fossilifère de sable très mal trié. Cette deuxième unité est peu stratifiée.

Elle est coiffée d'une troisième unité de matériel glaciomarin, de plus de 10,5 m d'épaisseur par endroits, dont la structure est lenticulaire. Les sédiments varient latéralement de silto-argileux caillouteux à silto-caillouteux. La teneur en carbonates de la matrice varie entre $30,9 \%$ et $51,8 \%$. La quantité de coquillages varie beaucoup d'une lentille à l'autre, de même que le pourcentage de galets ( $5 \%$ à $20 \%$ ). Dans les lentilles où le pourcentage de galets est important, ces derniers sont sub-anguleux et striés; là où le pourcentage est faible, ils sont arrondis.

À certains endroits, sur la côte basse s'insère, à la surface de cette troisième unité glacio-marine, une quatrième unité; celle-ci, d'épaisseur variable, est silto-sableuse, caillouteuse et très lenticulaire. Le contact entre ces deux unités est graduel. La présence en très grande quantité de Mya truncata et de Mya pseudo-arenaria montrent un milieu de vie plus favorable à la faune marine que lors de la sédimentation du matériel glacio-marin sous-jacent. Le pourcentage de galets est supérieur à $30 \%$ et la grande majorité d'entre eux sont sub- arrondis. La teneur en carbonates est relativement élevée pour ce type de matériel, soit de $55,2 \%$ à $73,4 \%$. Au-dessus de ces dépôts glacio-marins, on passe de façon abrupte à la phase d'exondation représentée par une unité de sédiments de plage. Cette dernière comporte plus de $4 \mathrm{~m}$ de lamelles de sable graveleux et de gravier sableux.

\section{CORRÉLATIONS ET CHRONOLOGIES DES SÉQUENCES DE LITHOFACIĖS}

\section{LES UNITÉS GLACIAIRES ET GLACIO-MARINES}

La corrélation entre les différentes colonnes stratigraphiques (fig. 3) se fait à l'aide des données sédimentologiques (tabl. I) et des datations ${ }^{14} \mathrm{C}$ (tabl. II) en relation avec deux dépôts de référence: le till brun pâle identifié sur presque tout l'ensemble du territoire et le till gris pâle fossilifère du secteur en gradins daté de $30000 \pm 1200$ ans BP (UQ-514) et de $29060 \pm 1050$ ans BP (UQ-510) (voir f8, fig. 3).

Le till gris pâle du secteur de gradins (fig. 3 , colonne B) est mis en corrélation avec les dépôts glacio-marins à la base des successions d'unités stratigraphiques dans les vallées principales en aval de la confluence avec les vallées étroites (fig. 3, colonnes $\mathrm{E}$ et $\mathrm{F}$ ). Ce till est aussi mis en corrélation avec l'unité glacio-marine inférieure de la zone côtière (fig. 3 , colonne $\mathrm{H}$ ). En effet, la date au ${ }^{14} \mathrm{C}$ des dépôts glacio-marins des vallées principales est de $36000 \pm 3500$ ans BP (UQ553) (voir f4, fig. 3); celle des dépôts glacio-marins du secteur côtier est de $28100 \pm 200$ ans BP (UQ-509) (voir f3, fig. 3). Par ailleurs, la corrélation entre ces différentes unités glaciomarines est établie à partir de trois critères: la structure lenticulaire, la teneur relativement faible en carbonates et la position stratigraphique.

De plus, la présence, dans le till gris pâle, de fragments de coquillages, ainsi qu'une teneur en argile un peu plus élevée, suggère que ce dernier provient probablement de l'érosion d'un sédiment marin d'eau profonde. La prise en charge de ce matériel marin a pu se faire à deux endroits: dans le détroit de Jacques-Cartier ou dans les bassins-versants adjacents au secteur de gradins. La deuxième hypothèse semble plus plausible, car une masse de glace abordant le nord de l'île provoque certainement un affaissement isostatique de l'ensemble du secteur et, étant donné la hauteur du niveau marin autour de 36000 ans BP (MILLIMAN et EMERY, 1968), les bassins ont sûrement été inondés.

Si l'unité déformée constituée de sédiments de transgression marine, se trouvant à la confluence des vallées étroites et principales (fig. 3 , colonne D), a été mise en place lors de cette inondation, elle peut être mise en corrélation avec le till gris pâle, comme étant en partie la source du till. Plusieurs éléments appuient cette corrélation: des graviers d'exondation couvrant directement les sédiments glacio-marins des vallées principales jusqu'au niveau actuel (fig. 3, colonnes $E$ et $F$ ) et la courbe de variation du niveau marin de MILLIMAN et EMERY (1968). La présence de ces graviers d'exondation signifie que, postérieurement à la récurrence glaciaire, il y a eu un relèvement isostatique jusqu'au niveau de la mer à cette époque. La courbe de variation du niveau marin démontre 
TABLEAU ॥

Description des datations au ${ }^{14} \mathrm{C}$

\begin{tabular}{|c|c|c|c|c|c|}
\hline $\begin{array}{l}\text { Localisation } \\
\text { stratigraphique } \\
\text { (voir fig. 3) }\end{array}$ & $\begin{array}{c}\mathrm{N}^{\circ} \text { de } \\
\text { laboratoire }\end{array}$ & Âge BP & Matériel daté & $\begin{array}{l}\text { Altitudes } \\
\text { (m) }\end{array}$ & Sédiments \\
\hline \multirow[t]{2}{*}{ f1 } & UQ-515 & $11950 \pm 150$ & coquillages & $5-10$ & $\begin{array}{l}\text { glacio- } \\
\text { marin }\end{array}$ \\
\hline & UQ-738 & $12190 \pm 170$ & coquillages & $2-3$ & $\begin{array}{l}\text { glacio- } \\
\text { marin }\end{array}$ \\
\hline \multirow[t]{4}{*}{ †2 } & UQ-505 & $13200 \pm 200$ & coquillages & $5-10$ & $\begin{array}{l}\text { glacio- } \\
\text { marin }\end{array}$ \\
\hline & UQ-502 & $13570 \pm 200$ & coquillages & $5-10$ & $\begin{array}{l}\text { glacio- } \\
\text { marin }\end{array}$ \\
\hline & UQ-496 & $13250 \pm 400$ & coquillages & $5-10$ & $\begin{array}{l}\text { glacio- } \\
\text { marin }\end{array}$ \\
\hline & UQ-551 & $14500 \pm 800$ & coquillages & $5-10$ & $\begin{array}{l}\text { glacio- } \\
\text { marin }\end{array}$ \\
\hline f3 & UQ-509 & $28100 \pm 200$ & coquillages & $3-5$ & $\begin{array}{l}\text { glacio- } \\
\text { marin }\end{array}$ \\
\hline$f 4$ & UQ-553 & $36000 \pm 3500$ & coquillages & $1-2$ & $\begin{array}{l}\text { glacio- } \\
\text { marin }\end{array}$ \\
\hline \multirow[t]{2}{*}{ f5 } & UQ-512 & $13100 \pm 150$ & coquillages & 14 & $\begin{array}{l}\text { marin d'eau } \\
\text { profonde }\end{array}$ \\
\hline & UQ-712 & $13400 \pm 140$ & coquillages & $12-14$ & $\begin{array}{l}\text { marin d'eau } \\
\text { profonde }\end{array}$ \\
\hline f6 & UQ-493 & $12400 \pm 140$ & coquillages & 18 & prodeltaïque \\
\hline \multirow[t]{3}{*}{$\uparrow 7$} & UQ-508 & $12080 \pm 280$ & coquillages & 18 & prodeltaiqque \\
\hline & UQ-499 & $12000 \pm 190$ & coquillages & 18 & prodeltaïque \\
\hline & UQ-498 & $11700 \pm 270$ & coquillages & 23 & prodeltaĩque \\
\hline \multirow[t]{2}{*}{ f8 } & UQ-510 & $29060 \pm 1050$ & coquillages & 113 & till \\
\hline & UQ-514 & $30000 \pm 1200$ & coquillages & 90 & till \\
\hline
\end{tabular}

qu'à partir de 35000 BP le niveau eustatique mondial ne fit que baisser, lentement jusqu'à 22000 ans BP, puis rapidement par la suite pour atteindre, vers 16000 ans BP, un seuil minimal (DUBOIS et al., 1983). Ces données nous montrent qu'il est peu probable qu'entre les deux récurrences glaciaires, après l'exondation du territoire, la mer inonda celui-ci à une altitude supérieure à $75 \mathrm{~m}$ au même moment que l'abaissement du niveau eustatique mondial.

En fonciton des énoncés précédents, l'unité de sédiments fluviatiles sous les sédiments de transgression marine (fig. 3 , colonne D) est probablement contemporaine de l'unité de sédiments prélittoraux mise en place directement sous l'unité inférieure de matériel glacio-marin du secteur côtier (fig. 3, colonne $\mathrm{H}$ ).

Le matériel fluvio-glaciaire surmontant le matériel glaciomarin des vallées principales (fig. 3, colonnes $E$ et F) est en corrélation avec une unité glacio-marine très caillouteuse mise en place sur la côte (fig. 3, colonne $\mathrm{H}$ ) au-dessus de l'unité glacio-marine inférieure. Ce matériel glacio-marin caillouteux implique un contact constant entre la glące et la mer, du moins sur les côtes basses, avant la mise en place des lits glacio-marins supérieurs silto-argileux (fig. 3, colonne $\mathrm{H}$ ) datés de $13200 \pm 200$ ans BP (UQ-505), $13570 \pm 200$ ans BP (UQ-502), $13250 \pm 400$ ans BP (UQ-496) et $14500 \pm$
300 ans BP (UQ-551) (voir f2, fig. 3). On a établi une corrélation entre ce matériel glacio-marin supérieur et le till brun pâle, ce dernier correspondant à une importante avancée glaciaire. La corrélation a été faite en fonction de la position stratigraphique des deux unités.

On a aussi établi une corrélation entre les tills bruns pâles représentés dans les colonnes $A, A^{\prime}, B, C, E, F$ et $G$ en fonction de la granulométrie, de l'étendue et de l'épaisseur des placages. Dans le secteur aval des vallées principales, on a associé ce till à un matériel glacio-marin caillouteux (fig. 3, colonne F). Le matériel glacio-marin supérieur est recouvert en partie par un troisième till, un till d'ablation, qui ne correspond à aucun autre matériel de la région à l'étude.

\section{LES UNITÉS MARINES ET D'EXONDATION}

Pour compléter la corrélation des successions d'unités stratigraphiques, il reste les unités relatives à la sédimentation marine et à l'exondation du territoire. Dans les vallées principales, deux unités marines d'eau profonde sont corrélatives (colonnes $\mathrm{E}$ et $\mathrm{F}$ ). La base de ces séquences est datée de $13100 \pm 150$ ans BP (UQ-512) et de $13400 \pm 140$ ans BP (UQ-712) (voir f5, fig. 3) à une altitude de $15 \mathrm{~m}$. À plus de $40 \mathrm{~m}$ d'altitude, l'unité de sédiments prodeltaïques est suivie d'un faciès de transgression marine (fig. 3 , colonne E) qui 
correspond chronologiquement au faciès de sédiments marins d'eau profonde mis en place plus en aval (fig. 3, colonne F). Dans les vallées principales, des unités de type prodeltaïque surmontent les unités marines d'eau profonde jusqu'à $75 \mathrm{~m}$ d'altitude. Ces sédiments prodeltaïques sont tous corrélatifs: par exemple, à $20 \mathrm{~m}$ d'altitude, ils sont datés de $12400 \pm$ 140 ans BP (UQ-493), $12080 \pm 280$ ans BP (UQ-508), $12000 \pm 190$ ans BP (UQ-499) et $11700 \pm 270$ ans BP (UQ-498) (voir f6 et f7, fig. 3).

Dans une coupe de la rivière Jupiter, vers $40 \mathrm{~m}$ d'altitude (fig. 3, colonne E), des sédiments prodeltaïques ont été déformés par une récurrence glaciaire. Cet événement correspond, sur la côte basse, à un changement observé dans la texture des dépôts glacio-marins (fig. 3 , colonne $\mathrm{H}$ ) : le matériel glacio-marin supérieur silto-argileux est couvert à certains endroits par un matériel glacio-marin silto-sableux daté de 12000 ans BP (UQ-515 et 738) (voir f1, fig. 3). Ces deux unités peuvent être mises en corrélation en raison de leur correspondance chronologique et stratigraphique.

Pour terminer, les unités fluviales d'exondation des vallées étroites (fig. 3, colonne C) et des vallées principales (fig. 3 , colonnes $\mathrm{D}, \mathrm{E}$ et $\mathrm{F}$ ) correspondent aux unités de sédiments littoraux (fig. 3, colonnes $G$ et $H$ ) des secteurs côtiers interfluviaux.

\section{HISTOIRE DU QUATERNAIRE}

La corrélation des successions d'unités stratigraphiques permet de reconstituer les milieux sédimentaires et d'établir les interrelations spatio-temporelles (GRATTON et al., 1983) (fig. 4).

L'histoire du Quaternaire supérieur que nous décrivons commence dans un milieu de sédimentation marine en phase transgressive. Cette interprétation est faite en fonction d'une unité de sédiments prélittoraux mise en place sur des sables caillouteux dans le secteur de la côte basse au niveau actuel de la mer et à partir d'une unité de sédiments fluviaux, qui passe graduellement à une unité de sédiments marins d'eau profonde à une altitude d'environ $75 \mathrm{~m}$. La présence de galets striés au sommet de cette dernière unité et la faible teneur en carbonates du matériel suggèrent que l'unité a été probablement mise en place en milieu glacio-marin proximal. Effectivement, la glace vient par la suite perturber les sédiments, mais ne réussit pas à s'introduire plus profondément dans les parties inondées des vallées principales (fig. 4 et 5). Dans ces vallées, la sédimentation glacio-marine s'est faite sous $70 \mathrm{~m}$ d'altitude. La variation de la texture des sédiments glacio-marins est liée à la dynamique d'ablation de la glace, de même qu'à la proximité du front glaciaire. Ces sédiments, datés d'environ 36000 ans BP, sont corrélatifs et sont reliés à une récurrence glaciaire du Wisconsinien moyen (fig. 4). Par contre, la glace ancrée a pu progresser dans les secteurs en gradins et mettre en place, vers 30000 ans BP, le till gris pâle provenant en grande partie de l'érosion de sédiments marins fossilifères. Le front glaciaire s'arrête à la limite de la zone côtière haute et laisse un matériel glacio-marin sur les côtes basses vers 28000 ans BP (fig. 4 et 5).
À partir de ce moment, la glace s'amincit sur les interfluves et un matériel glacio-marin caillouteux est mis en place. Cette ablation partielle de la glace occasionne un réajustement isostatique et une exondation des terres. Dans les vallées principales, les eaux de fonte abondantes laissent des sédiments fluvio-glaciaires dans une mer en régression, et ce, jusqu'au niveau actuel de la mer. À ce moment, une récurrence mineure locale de la glace se produit, suivie d'un recul, avec pour résultat une interstratification de till et de matériaux fluvioglaciaires (fig. 4).

Cette oscillation est suivie d'une récurrence glaciaire majeure qui couvre la totalité du territoire et met en place le till brun pâle typique de l'événement (fig. 4). Cette récurrence est synchrone avec une seconde invasion marine. La glace est ancrée partout, sauf au-dessous de $20 \mathrm{~m}$ d'altitude (fig. 4 et 6). Un vêlage intense du front glaciaire limite l'extension de la nappe de glace. En dessous de $20 \mathrm{~m}$ d'altitude, dans les vallées principales, se met en place un matériel glaciomarin caillouteux et, sur les côtes basses, un matériel glaciomarin silto-argileux corrélatif, vers 13500 ans BP.

À cette époque, des fluctuations de l'écoulement glaciaire ou du niveau marin provoquent, dans les vallées principales, une invasion mineure locale de la masse de glace (fig. 4). Cet événement permet la mise en place d'un till d'ablation à $20 \mathrm{~m}$ d'altitude, au-dessus du matériel glacio-marin caillouteux.

Par la suite, dans les vallées principales encore, l'invasion marine provoque un vêlage de plus en plus intense du front glaciaire; ceci est confirmé par la présence de sédiments marins massifs d'eau profonde. Cette incursion de la mer de Goldthwait représente le début du postglaciaire. La base de cette séquence marine d'eau profonde est datée de 13000 ans BP.

Le retrait glaciaire s'accélère, surtout dans les vallées principales. À partir de $40 \mathrm{~m}$ d'altitude, le contact entre l'eau de fonte et la mer en transgressison rapide occasionne une sédimentation de matériel prodeltaïque suivie d'une sédimentation marine d'eau profonde. La glace abandonne les vallées principales, s'amincit dans l'ensemble du territoire et ne subsiste que sur les interfluves et dans les vallées étroites. Cette fonte rapide provoque le relèvement isostatique et le début de la régression marine finale. La mer a commencé à se retirer à partir d'une altitude d'au moins $75 \mathrm{~m}$, comme le démontre la présence de sédiments prodeltaïques mis en place, à cette altitude, au cours du processus de régression.

Selon PAINCHAUD, DUBOIS et GWYN $(1982,1984)$, la régresion marine est aussi synchrone avec l'abandon d'une calotte anticostierne résiduelle. Dans les secteurs côtiers interfluviaux, la masse glaciaire, tout en s'amincissant, recule lentement. Quand le niveau marin atteint $20 \mathrm{~m}$ d'altitude, vers 12000 ans BP, un réajustement glacio-dynamique de la calotte se produit. En effet, lors de la récurrence majeure, le vêlage intensif de la masse glaciaire dans une mer profonde a bloqué son extension; la régression marine a donc provoqué un réajustement gravitationnel de la calotte en fonction de la nouvelle limite marine (fig. 4). La récurrence qui en a découlé, et que l'on nomme anticostienne, perturbe, à $40 \mathrm{~m}$ d'altitude dans la vallée de la rivière Jupiter, les sédiments prodeltaïques 


\section{UNITĖ GÉOMORPHOLOGIQUE}

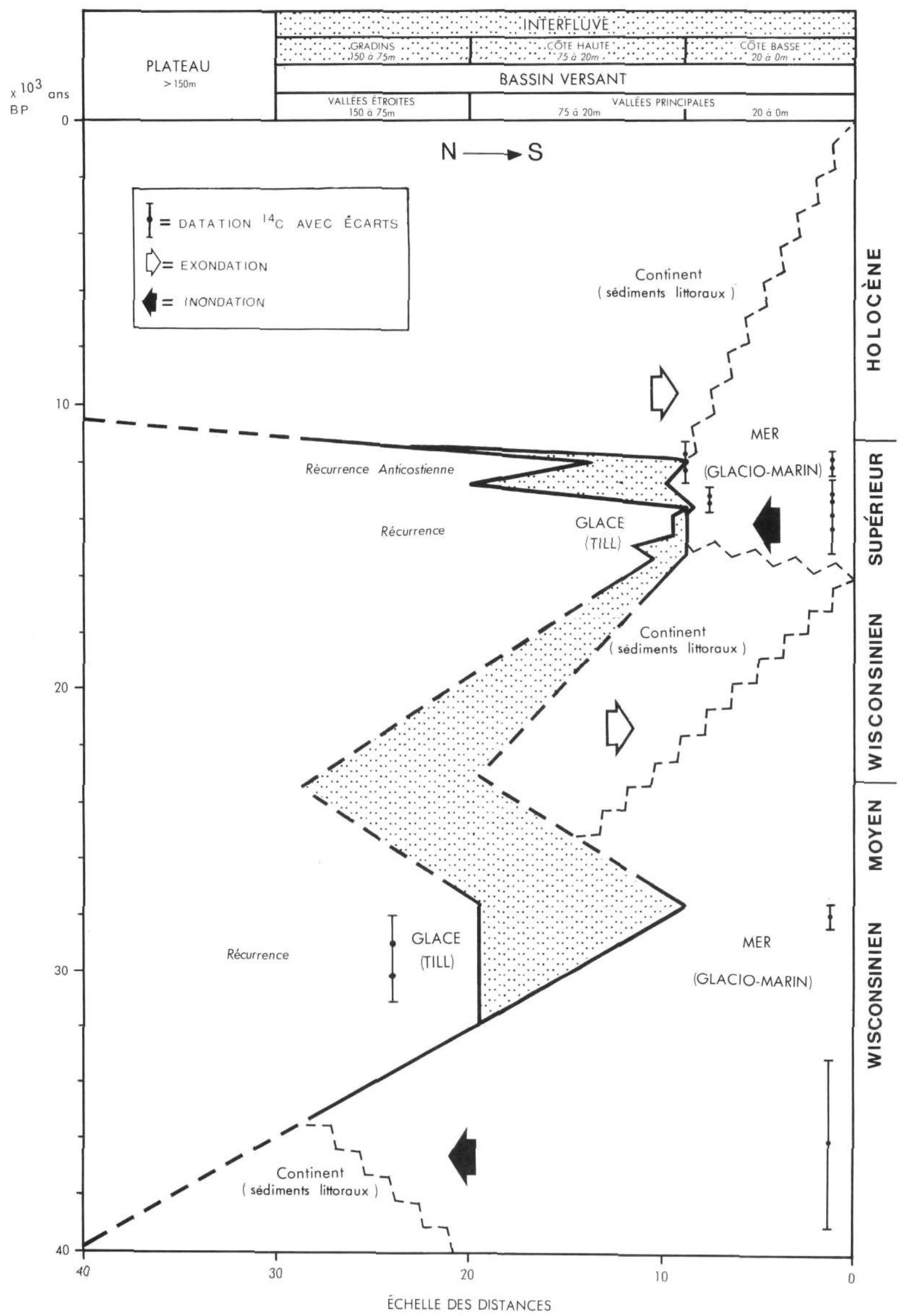

ÉCHELLE DES DISTANCES

FIGURE 4. Schéma spatio-temporel des événements glaciaires en distinguant la limite glaciaire sur les interfluves et dans les bassinsversants; les niveaux marins sont approximatifs.
The space-time sketch of glacial events with reference to the glacial limits on the interfluves and the river valleys; the marine limits are approximative. 


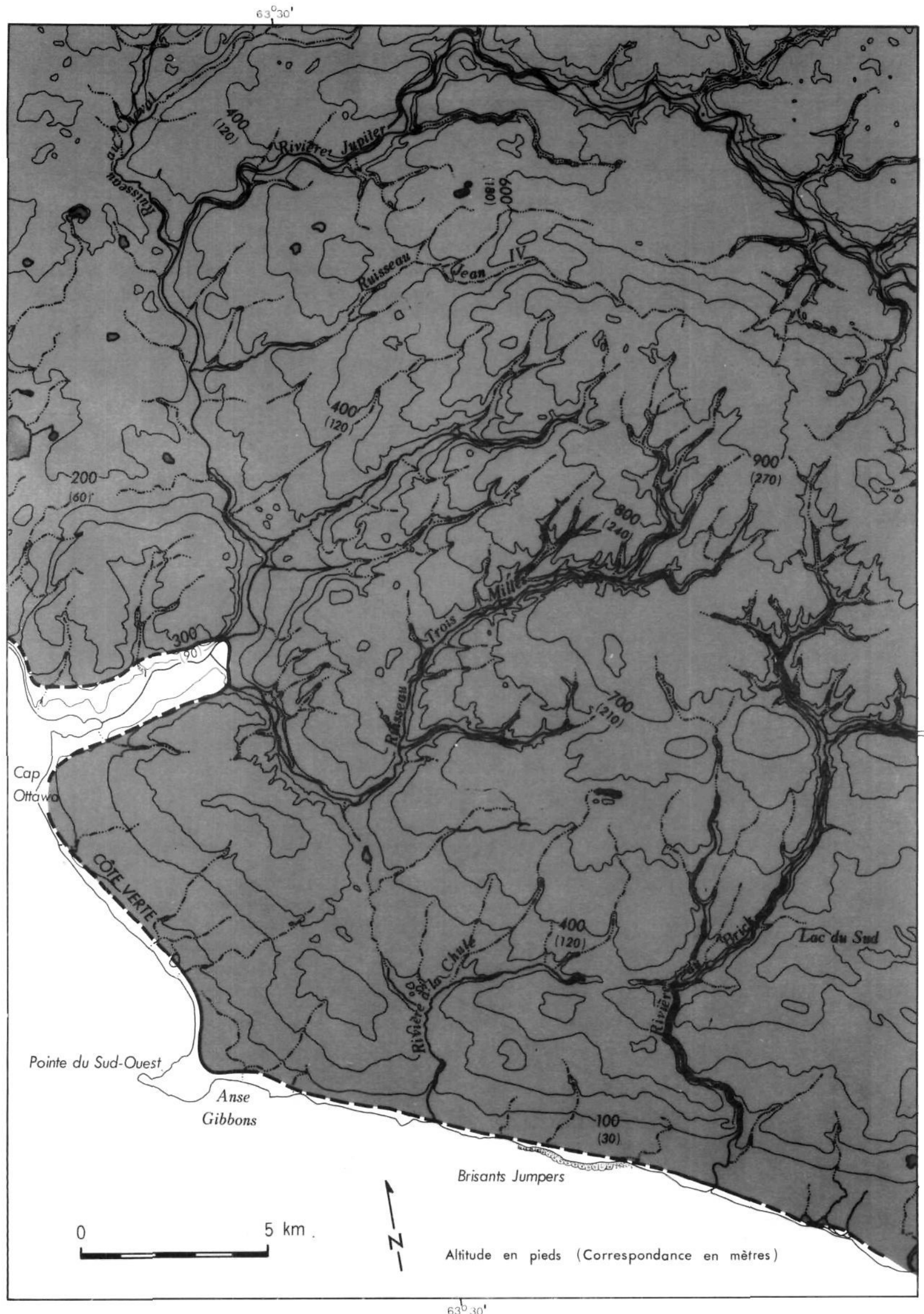

FIGURE 5. Limite de la glace ancrée au Wisconsinien moyen.

Mid-Wisconsinan grounded-ice limit. 


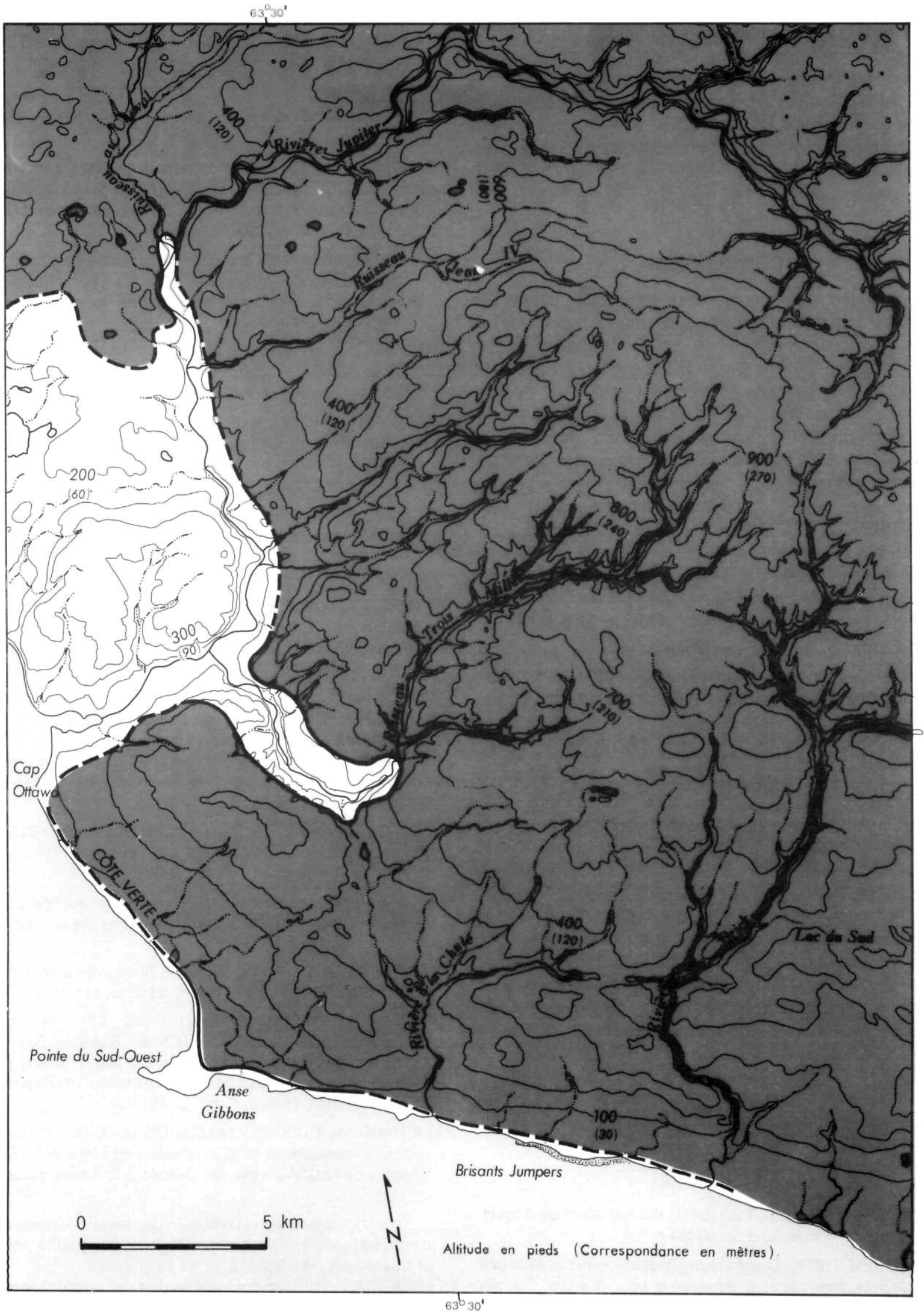

FIGURE 6. Limite de la glace ancree au Wisconsinien supérieur. Late-Wisconsinan grounded-ice limit. 
de la phase de régression et met en place, sur les côtes basses, un matériel glacio-marin sablo-silteux daté d'environ 12000 ans BP.

La dernière récurrence est suivie d'un amincissement progressif de la calotte et laisse des épandages importants de matériel fluviatile dans les vallées. Sur les interfluves côtiers, au fur et à mesure du relèvement isostatique, une sédimentation littorale témoigne de la régression de la mer de Goldthwait jusqu'au niveau actuel.

\section{CONCLUSION}

La paléogéographie quaternaire du centre-sud de l'île d'Anticosti nous permet d'appuyer, en partie, l'hypothèse minimaliste en confirmant que le golfe du Saint-Laurent n'a pas été englacé depuis 36000 ans BP. En effet, il ressort de notre étude deux conclusions importantes: d'abord, l'englacement de l'île, au Wisconsinien moyen et au Wisconsinien supérieur, par deux récurrences majeures de l'inlandsis laurentidien; de plus, l'existence d'une récurrence mineure locale qui a précédé et suivi l'événement du Wisconsinien supérieur. Nous confirmons aussi la récurrence anticostienne présentée par PAINCHAUD, DUBOIS et GWYN $(1982,1984)$. Deuxièmement, nous démontrons qu'avant et durant les deux récurrences majeures, la mer a submergé la côte sud de l'île; le vêlage intensif des masses de glace dans une mer de plus en plus profonde a empêché leur extension dans le golfe.

La détermination de la limite sud de l'inlandsis laurentidien au Wisconsinien moyen et supérieur contribuera à résoudre certains litiges liés au modèle d'englacement d'autres côtes du golfe.

\section{REMERCIEMENTS}

L'aide financière a été fournie par le fonds FCAC du Québec (EQ 102), le CRSNG (A-4250) et la Commission géologique du Canada (249-4-80, 244-4-81, 144-4-82). Nous remercions également les membres du projet Anticosti pour leur participation aux travaux de terrain et pour les judicieux conseils qu'ils nous ont donnés. Nous tenons aussi à remercier Pierre Pitre et Michel Fournier du ministère du Loisir, de la Chasse et de la Pêche à Port-Meunier, pour leur aide technique. Nous remercions Germain L. Tremblay pour l'analyse sédimentologique de plusieurs échantillons, Diane Langlois, Richard Mailhot et François Charron pour la réalisation des figures et finalement, Danielle Marceau, Jean-Serge Vincent, la rédaction de la revue et un lecteur anonyme qui ont largement aidé à améliorer le texte de l'article.

\section{RÉFÉRENCES}

DENTON, G.H. et HUGUES, T.J. (1981): The last Great lce Sheets, New York, John Wiley and Sons, $484 \mathrm{p}$.

DUBOIS, J.M.M. (1979): Environnements quaternaires et évolution littorale d'une zone côtière en émersion en bordure sud du Bouclier Canadien: la Moyenne Côte Nord du Saint-Laurent, Thèse de Ph.D., Univ. d'Ottawa, 754 p.
DUBOIS, J.M.M., PAINCHAUD, A., GWYN, Q.H.J. et GRATTON, D. (1983): Histoire des mouvements de la croûte terrestre depuis 35000 ans dans le nord du golfe du Saint-Laurent, $51^{\circ}$ congrès de l'ACFAS, Trois-Rivières, 25-27 mai, texte de communication.

FLINT, R.R. (1943): Growth of the North American ice sheet during the Wisconsin Age, Geological Society of America Bulletin, vol. 54 , $\mathrm{n}^{\circ} 3$, p. 325-367.

GRANT, D.R. (1976): Late Wisconsinan ice limits in the Atlantic Provinces with particular reference to Cape Breton Island, Nova Scotia, in Report of Activities, Part C, Geological Survey of Canada, Paper 76-1c, p. 289-292.

(1977): Glacial style and ice limits, the Quaternary stratigraphic record, and changes of land and ocean level in the Atlantic provinces, Canada, Géographie physique et Quaternaire, vol. 31, $n^{\text {os }} 3-4$, p. 247-260.

GRATTON, D. GWYN, Q.H.J. et DUBOIS, J.M.M. (1982): Les séquences fluviales, marines et glacio-marines, centre de l'île d'Anticosti, Annales de l'ACFAS, vol. 49, p. 128.

GRATTON, D., GWYN, Q.H.J., DUBOIS, J.M.M. et PAINCHAUD, A. (1983): La limite glaciaire au Wisconsinien moyen et tardif, Annales de l'ACFAS, vol. 50, p. 122.

GWYN, Q.H.J., DUBOIS, J.M.M. et GRATTON, D. (1983): Late Wisconsinan ice marginal geomorphology and sedimentation in the coastal zone, northern Gulf of St. Lawrence, Geological Society of America, N.E. section, Kiamesha Lake, N.Y., Abstracts with programs, vol. 15, n 3, p. 194.

GWYN, Q.H.J., DUBOIS, J.M.M., PAINCHAUD, A. et GRATTON, D. (1982): Sédimentologie post-glaciaire de la plateforme continentale émergée, île d'Anticosti, Québec, $11^{\circ}$ Congrès international de sédimentologie, Univ. McMaster, Hamilton, Abstracts with programs, p. 159.

GWYN, Q.H.J., GRATTON, D. et DUBOIS, J.M.M. (1983): The development of late Quaternary lithofacies sequences and their chronological control, northern Gulf of St. Lawrence, Correlation of Quaternary Chronologies Symposium, York Univ., Abstracts with programs, p. 64-65.

GWYN, Q.H.J., GRATTON, D., PAINCHAUD, A., DAIGNEAULT, R.A. et DUBOIS, J.M.M. (1982): Les dépôts glacio-marins, île d'Anticosti, Annales de l'ACFAS, vol. 49, p. 137.

IVES, J.D. (1978): The maximum extend of the Laurentide ice sheet along the east coast of North America during the last glaciation, Arctic, vol. 31, $\mathrm{n}^{\circ} 1$, p. 24-53.

MILLIMAN, J.D. et EMERY, K.O. (1968): Sea Levels during the past 35000 years, Science, vol. 162, no 3858, p. 1121-1123.

OSTERMAN, L.E. et ANDREWS, J.T. (1983): Changes in glacialmarine sedimentation in core HU77-159, Frobisher Bay, Baffin Island, N.W.T.: A record of proximal, distal, and ice-rafting glacialmarine environnements, in, Glacial-Marine Sedimentation, B.F. Molnia, édit. Plenum Press, p. 451-494.

PAINCHAUD, A., DUBOIS, J.M.M. et GWYN, Q.H.J. (1982): Les systèmes morainiques de la zone ouest de l'île d'Anticosti, 50 Congrès de l'ACFAS, Univ. du Québec à Montréal, texte de la communication.

(1984) : Déglaciation et émersion des terres de l'ouest de l'île d'Anticosti, golfe du Saint-Laurent, Québec, Géographie physique et Quaternaire, vol. 38, n० 2, p. 43-111.

PÉTRYK, A. A. (1979): Stratigraphie revisée de l'île d'Anticosti, Min. de l'Énergie et des Ressources, Direction générale de l'énergie, Service de l'exploration, Québec, DPV-711, 24 p. 